Sharif University of Technology
Scientia Iranica
SCIENTIA $\quad \begin{gathered}\text { Transactions D: Computer Science \&s Engineering and Electrical Engineering } \\ \text { http://scientiairanica.sharif.edu }\end{gathered}$

\title{
TQCAsim: An accurate design and essential simulation tool for ternary logic quantum-dot cellular automata
}

\author{
A. Navidi ${ }^{a}$, R. Sabbaghi-Nadooshan ${ }^{\mathrm{b}, \mathrm{c}, *}$, and M. Dousti ${ }^{\mathrm{a}}$ \\ a. Department of Electrical and Computer Engineering, Science and Research Branch, Islamic Azad University, Tehran, Iran. \\ b. Department of Electrical Engineering, Central Tehran Branch, Islamic Azad University, Tehran, Iran. \\ c. School of Computer Science, Institute for Research in Fundamental Sciences (IPM), Tehran, Iran.
}

Received 4 May 2019; received in revised form 20 November 2020; accepted 8 March 2021

\section{KEYWORDS}

Quantum-dot Cellular Automata (QCA);

Ternary logic;

TQCAsim;

TQCA;

Simulator.

\begin{abstract}
Having a reliable simulation tool for evaluating the performance of each design is indispensable. Designing Multiple-Valued Logic (MVL) systems helps overcome the limitations associated with binary systems. Quantum-dot Cellular Automata (QCA) is a technology that can be substituted for Complementary Metal-Oxide-Semiconductor (CMOS) in MVL designs. This paper represents an exquisite software platform for designing and simulating circuits that are restricted to Ternary QCA (TQCA). Working with TQCAsim is so convenient because it can run on both Windows or Linux-based computers. It has a tenacious simulation engine that can ensure precise results. This tool shows the results in graphical formats. Moreover, designers can easily layout their ternary QCA designs using various sets of CAD tools. In this paper, the ternary model of QCA and its energy calculation will be demonstrated. The simulation process is explained step by step. MIN, MAX, NOT, and XOR gates were simulated already by this software.

(C) 2022 Sharif University of Technology. All rights reserved.
\end{abstract}

\section{Introduction}

The Quantum-dot Cellular Automata (QCA) is one of the new developing technologies, which is a suitable candidate for Complementary metal-oxidesemiconductor (CMOS) replacement in the future [1,2]. Logic circuit designers had proposed their designs over the past two decades [3,4]. QCA cells (semiconductor and metallic) have been fabricated and examined. The fabrication of molecular QCA was a remarkable achievement for developers because it can operate at room temperature $[5,6]$. Plenty of creative designs have

\footnotetext{
*. Corresponding author. Tel.: +982144600047;

Fax: +982144600071

E-mail addresses: alireza.navidi@srbiau.ac.ir (A. Navidi);

r_sabbaghi@iauctb.ac.ir (R. Sabbaghi-Nadooshan);

m_dousti@srbiau.ac.ir (M. Dousti)
}

been proposed and published in the field of Binary QCA (BQCA) logic [7-13] and ternary [14-17].

Compacting data in fewer bits is achieved by modeling systems in Multiple-Valued Logic (MVL). A significant amount of data can be transferred in MVL systems compared with binary ones [18]. MVL designs can provide faster numerical analysis and arithmetic operations, but designing and simulating these types of systems is not so easy and needs its CAD tools [19].

Every innovative concept needs a particular way of determining its functionality. The ternary QCA lacked simulation tools. This work represents a tool that is capable of simulating complex ternary QCA circuits. In this paper, the functionality of the software would be clarified step by step. TQCAsim uses a fast simulation engine that makes the computation performed in a fraction of time. In other published work, only the ternary QCA circuits have been proposed without explaining the software structure [2022]. Section 2 presents the ternary QCA basics. Sec- 
tion 3 highlights the main functions of the simulator. Section 4 gives a benchmark for evaluating TQCAsim performance. Section 5 describes incoming plans that would make TQCAsim more thriving. At last, Section 6 concludes this paper.

\section{Ternary QCA basics}

The QCA is a flourishing technology that can carry out computations by Coulombic interaction, while CMOS is dependent on the electrical current [23,24]. A QCA cell is a square nanostructure with some quantum wells confining free electrons [25]. A certain number of quantum-dots have been embedded in each cell so that the mobile pair of electrons can tunnel between them. There are four quantum-dots in BQCA while only two in Ternary QCA (TQCA).

According to the Coulombic law, the two electrons will not be distributed in precarious situations among the quantum-dots, i.e., they reside furthest away from each other [25]. Figure 1 shows different modes of electrons positioning in a TQCA cell. A, B, C, D are stable modes (electrons are located at a far distance from each other due to the Coulomb repulsion effect), and the $\mathrm{X}$ modes are unstable.

TQCA has a polarization states that can be related to the logic values. However, there is a discrepancy between the number of polarization states in TQCA and the number of ternary logic values. There are four stable polarization states for TQCA cells (A, B, C, and D), which are equivalent to logic values $-1,+1,0$, and 0 , respectively (balanced ternary). So, there are two possible states (C and D) with an identic value (0). Figure 2 exhibits all four possible configurations of a TQCA cell.

The similarity between BQCA and TQCA is that
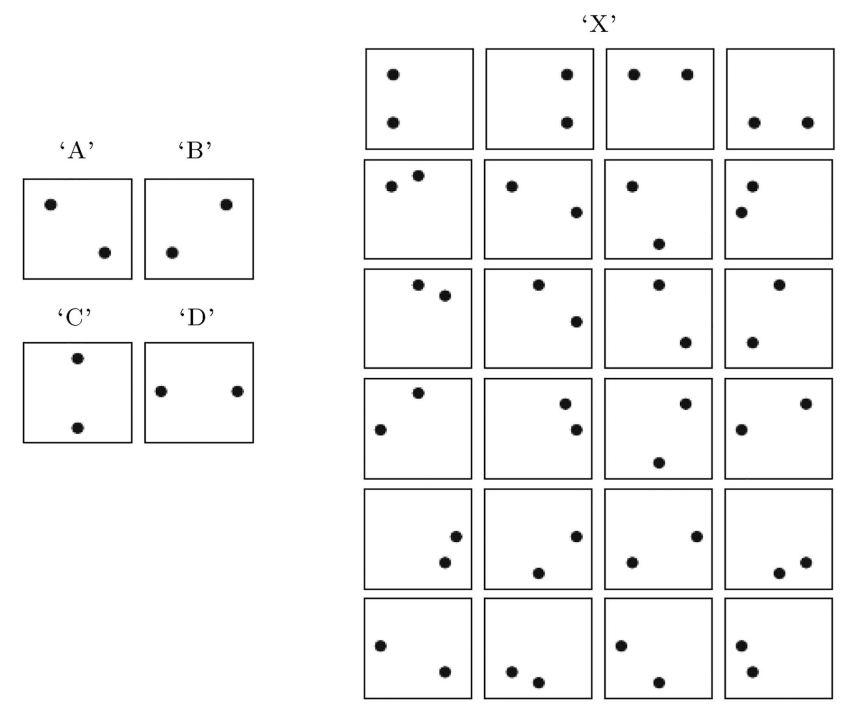

Figure 1. Different modes of electrons positioning in a TQCA cell.
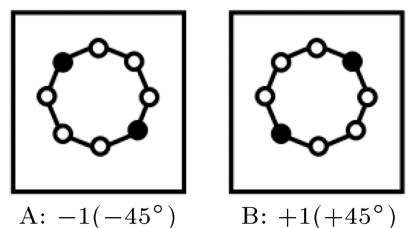

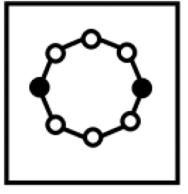

C: $0\left(0^{\circ}\right)$

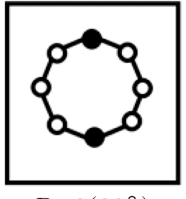

D: $0\left(90^{\circ}\right)$
Figure 2. Four possible configurations of a TQCA cell.

states $\mathrm{A}$ and $\mathrm{B}$ in the ternary mode are the same in binary. Also, the analogy between them in the clock issue is explicit. All four phases of the clock (switch, hold, release, and relax) in TQCA are the same in BQCA. These clocks are being used to push information from inputs to the outputs by modifying the cells' tunneling energy. The whole circuit is segmented into zones. Each zone is associated with one of the four clocks in sequence [26].

\subsection{Utilized TQCA cell}

As mentioned in Section 1, QCA implementation is already divided into four categories: metal [27], semiconductor [28], molecular [29,30], and magnetic [31]. The other advantage of molecular QCA rather than working at room temperature is that it can be fabricated at minute dimensions. Therefore, TQCAsim is based on this type of QCA implementation. Figure 3 shows the utilized TQCA cells in this work. However, TQCAsim can scale the cell's dimensions.

\subsection{Energy calculation}

TQCAsim is based on calculating external electrostatic energy between neighbor cells. It computes the distance between the electron's positions in adjacent cells. It is quite noticeable that two QCA cells could be adjacent in horizontal, vertical, or even diagonal directions. Figure 4 shows the distances between electrons of two adjacent cells if both of them remain in the ' $\mathrm{B}$ '

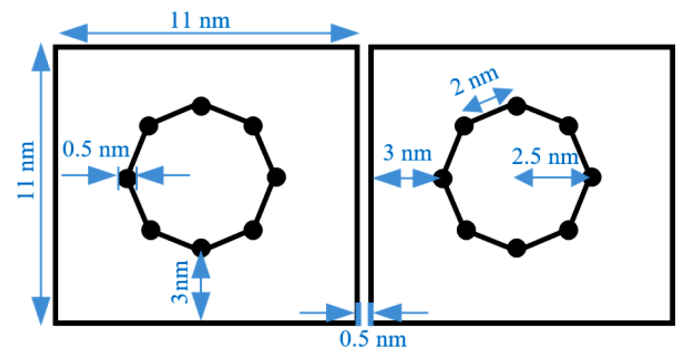

Figure 3. Utilized TQCA cells.

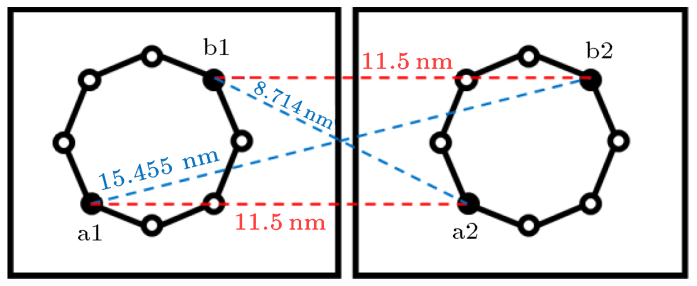

Figure 4. Distance between electrons of two adjacent cells in the 'B' polarization state. 
polarization state, i.e., it was assumed that both of the input (left one) and output (right one) cells had +1 $(+45)$ values.

Each cell affects the neighboring cells by means of external electrostatic energy. This energy can be attained from Eq. (1):

$$
E_{\text {external }}=\frac{1}{4 \pi \varepsilon_{0} \varepsilon_{r}} \sum_{i=0}^{m} \sum_{j=0}^{n} \frac{q_{i} q_{j}}{d_{i j}},
$$

where $\varepsilon_{0}$ and $\varepsilon_{r}$ are vacuum and substance relative permittivity coefficients, respectively. $q_{i}$ and the $q_{j}$ are the charges of dots in the cell, and $d_{i j}$ is the distance between the two. The external electrostatic energy calculation between the electrons of two adjacent cells in Figure 4 is as follows:

$$
\begin{aligned}
& E_{e 1}=E_{a 1-a 2}=2.005 \mathrm{e}-20 \mathrm{~J}, \\
& E_{e 2}=E_{b 1-b 2}=2.005 \mathrm{e}-20 \mathrm{~J}, \\
& E_{e 3}=E_{a 1-b 2}=1.492 \mathrm{e}-20 \mathrm{~J}, \\
& E_{e 4}=E_{b 1-a 2}=2.646 \mathrm{e}-20 \mathrm{~J} .
\end{aligned}
$$

The ultimate energy quantity would be obtained by subtracting the summation of similar named energies and the dissimilar named energies. Eq. (3) demonstrates the conclusive energy quantity for Figure 4.

$$
E_{t}=\left(E_{e 3}+E_{e 4}\right)-\left(E_{e 1}+E_{e 2}\right)=0.128 \mathrm{e}-20 \mathrm{~J} .
$$

All the other possible states with their final energy quantities are shown in Figure 5. The correct output state is the one with the minimum energy value among different states due to the final energy results in Figure 5. For example, if the input value is $+1(+45)$, the output value will be $+1(+45)$. Further investigations into diverse states with their energy calculation were documented [20].

\section{Simulation setup}

This project was written in C\# under Microsoft Visual Studio. The GUI interface of TQCAsim was developed using Windows Forms. Linux users may also use this tool by installing the Windows application runtime (EXE) like Wine and .NET Framework runtime libraries. TQCAsim uses the .NET library to generate graphical models. The objective of this project is to create an easy-to-use simulation and layout tool. The latest version of the software can be found in [32].

Innovative TQCA designs were documented as proof-of-concept experiments [20-22]. The other authors have proposed ternary architectures using TQCAsim and confirmed the correct operation of our CAD tool $[33,34]$.

Figure 6 illustrates the flowchart of the program in a general schema. In the following, the functionality of each section is explained.

\subsection{Modeling scheme}

A $16 \times 32$ cell matrix is considered to design logic circuits in the current version of the software layout environment. A TQCA circuit may consist of four different types of cells:

- Input/output cell;

- Cell with fixed polarity;

- Intermediate cell;

- Interface cell.

Each type of cell is shown with a distinct color in the layout environment, i.e., the color of each one depends on the cell's type.

At first, the kind of each cell must be determined by the user. The simulator employs a relevant icon for each cell according to its type. Then, appropriate clocks should be assigned. The software would enter the simulation phase by pressing the simulation key. It

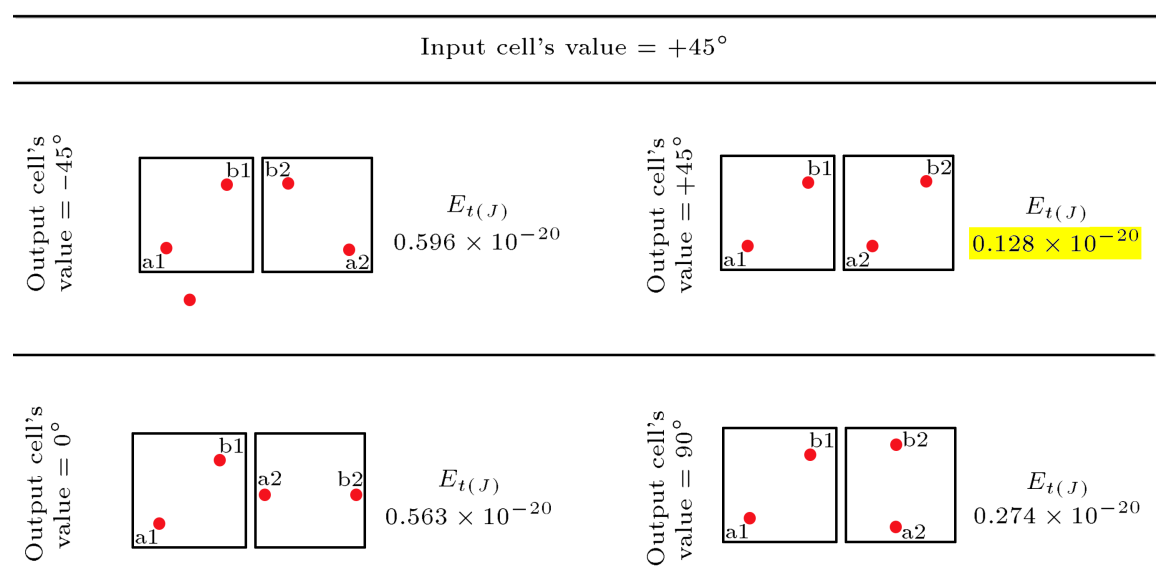

Figure 5. Energy calculation for two adjacent cells. 


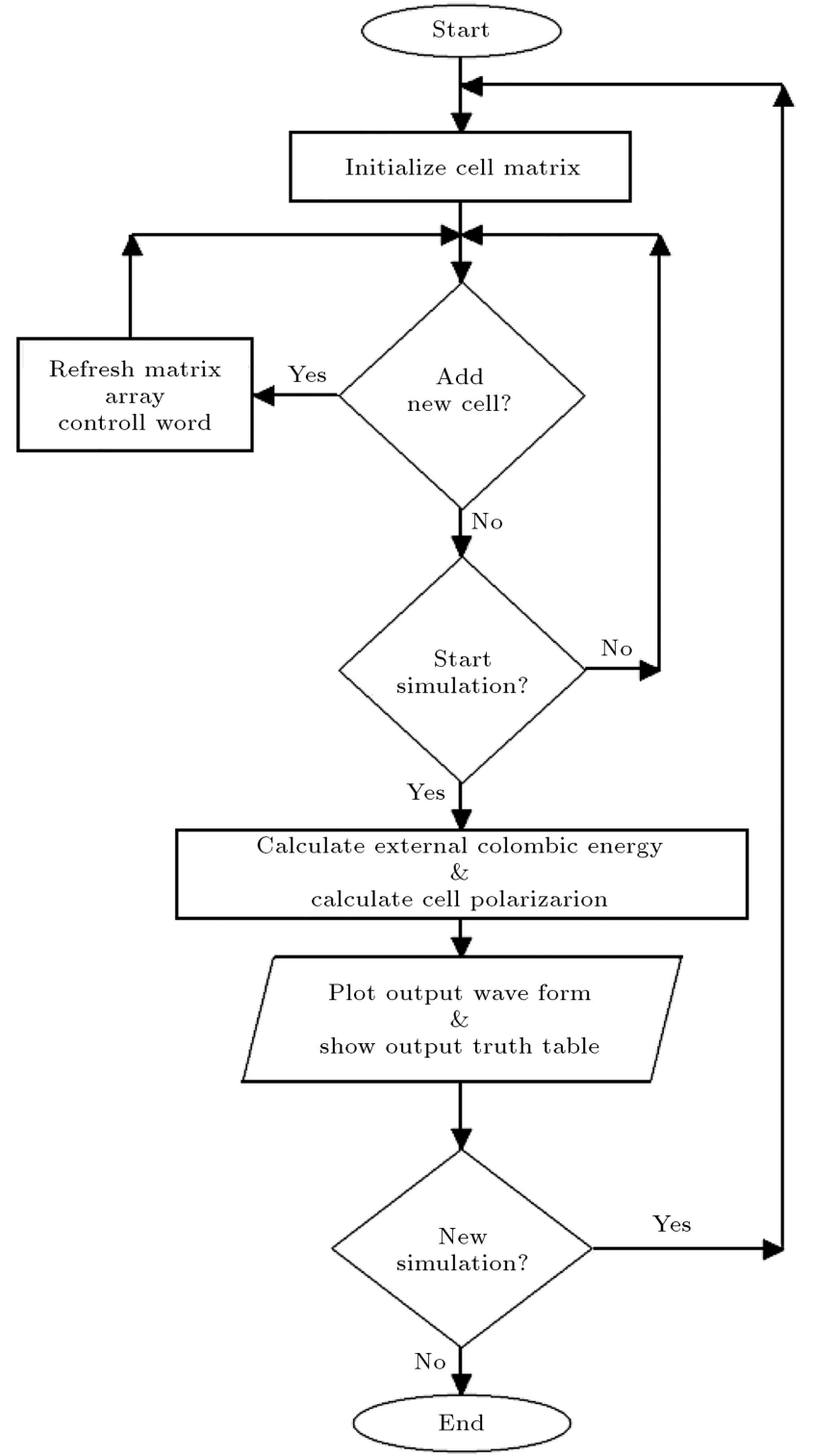

Figure 6. General flowchart of the simulator.

will consider all different possible values for inputs and will display the truth-table.

\subsection{Simulation process}

The polarity of the intermediate and output cells will be specified according to the energy calculations among cells at the simulation stage. The sequence of the simulation steps is listed below:

1. Calculating the number of input/output cells and saving their coordinates in an array of global type. The circuit's outputs should be computed and displayed in the truth-table with all possible combinations of inputs. Therefore, the number of input and output cells is counted and stored in a globaltype variable in the first step of the simulation.

2. Entering into the initializing loop of the input cells. At this point, three values of ternary $(0,-1,+1)$ would be replaced for each input cell. Subsequently, the output would be calculated for them. For example, for a circuit with two input cells, the simulator will calculate the output cells for nine different combinations.

3. Determining the priority of intermediate cells based on their positions and clock's phases. One of the most important steps is to determine the order of fixed-polarity cells' effect. As mentioned earlier, the polarity of the middle cells is affected by the cells with fixed polarity under the Coulomb interaction force. This effect is inversely related to the distance between them, i.e., the longer the distance between the cells yields less effect on each other. Therefore, the simulator considers the number of fixed-polarity cells around the surrounded cell with their distance from each other. The coordinates of the surrounded cell with its clock's phase are given as inputs to the calculator function. Therefore, numbers from 0 to 280 will be produced as a distribution priority output. Cells with fixed polarity adjacent to the target cell with one neighborhood interspace have the highest priority as Rank 5. Cells with fixed polarity adjacent to the target cell with two neighborhood interspaces are of lower priority as Rank 4 at the second round of ranking. The task of priority determination is performed for five neighborhood interspaces in the case of each cell. Figure 7 shows the priority values for one and two neighborhood interspaces. Thus, the maximum calculated priority for each cell is $280(40+64+72+64+40=280)$.

According to the QCA's clock principle, only cells with the same phase or -90 degrees shifted phase affect their neighbors. Therefore, the priority would be determined separately for cells with diverse clocks. The point is that cells' polarization with the same precedence will simultaneously be determined. For example, Figure 8 shows the calculated priority for a typical circuit. In this circuit, the highest priority is allotted to cell $\mathrm{C}$. Cells A and B have lower priorities.

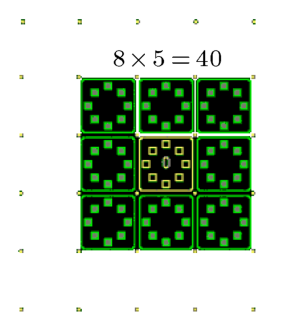

(a)

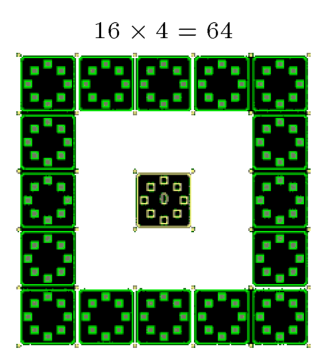

(b)
Figure 7. Priority values for (a) one and (b) two neighborhood interspaces. 


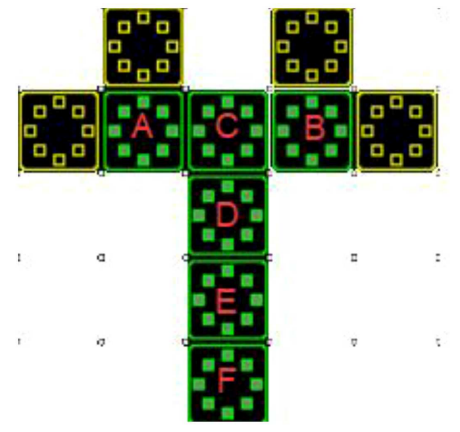

$A=(5+5+4+3)=17$ $B=(5+5+4+3)=17$ $C=(5+5+4+4)=18$ $D=(4+4+4+4)=16$ $E=(4+4+3+3)$ $F=(3+3+2+2)=10$

Figure 8. Calculating priority.

4. Specifying the intermediate cell's polarity according to its predefined priority. The next step is to specify the polarity for intermediate cells due to their priority from the highest down to the lowest one. Cell $\mathrm{C}$ is the first cell whose polarity is specified in Figure 8. Then, the polarity of cells A and B would be individually and without considering the effect of each other. The polarity of cells D, E, and $\mathrm{F}$ remains to be considered in the next steps. Polarity is assigned to the cells according to the energy calculation, as discussed in Section 2.

\subsection{Results display}

The truth table of the circuit will be plotted right after the calculation hierarchy ends. Figure 9 shows a sample circuit with its truth table. This software verifies the outputs for all possible input combinations. Figure 10 shows a snapshot of the TQCAsim while displaying the result. As can be seen, the tool displays the inputs and outputs by four different clock signals. TQCAsim's team attempted to form a user interface
Simulation result

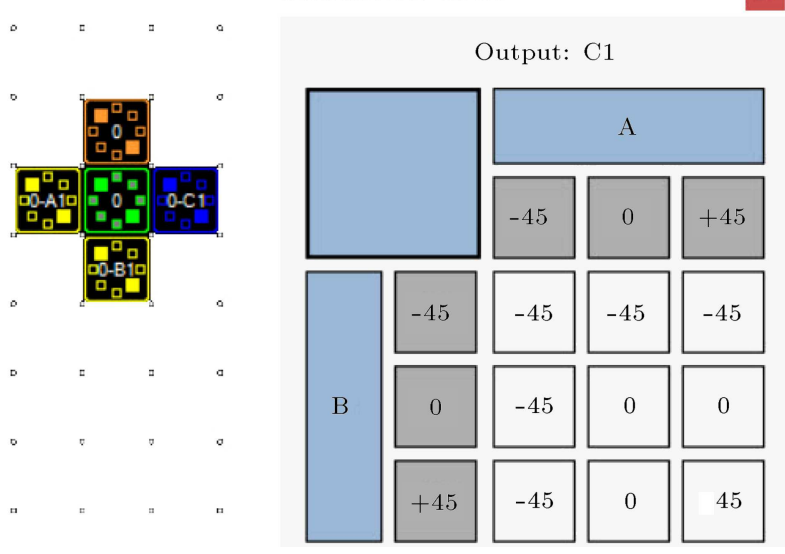

Figure 9. A sample circuit with its truth table.

similar to the QCADesigner [35] so that designers who had previously worked in the binary field would feel comfortable working with this software.

\section{Performance benchmark}

In this section, a benchmark is given for evaluating the performance of TQCAsim. We have tested this software on two different systems. System 1 has an obsolete CPU and operating system. On the other hand, System 2 has an advanced CPU and an up-to-date operating system. In this benchmark, three primary ternary gates (NOT, AND/OR, and Majority) were simulated '10' times in a row at a specified run time. Figure 11 shows the schematics of these gates. Table 1 comprises the benchmark results.

The TQCAsim needs only $10.8 \mathrm{MB}$ of free space on a drive. As can be seen from Table 1, TQCAsim has

Table 1. Benchmark results.

\begin{tabular}{|c|c|c|c|c|c|}
\hline & \multicolumn{2}{|c|}{ CPU } & \multirow{2}{*}{$\frac{\text { Memory }}{\text { Private }^{c}(k B)}$} & \multirow{2}{*}{$\begin{array}{c}\text { Run-time } \\
\text { (sec) }\end{array}$} & \\
\hline & Threads $^{\mathrm{a}}$ & $\operatorname{Avg}^{\mathbf{b}}$ & & & \\
\hline \multicolumn{6}{|l|}{ System 1} \\
\hline Processor: Intel ${ }^{\circledR}$ Core $^{\mathrm{TM}} 2$ DUO T8300@ $@ 2.4 \mathrm{GHz}$ & 6 & 3.22 & 40904 & 60 & Not \\
\hline Installed Memory: $4.00 \mathrm{~GB}$ & 7 & 3.13 & 42628 & 105 & And/or \\
\hline Operating System: Windows 7 & 7 & 4.57 & 43380 & 135 & Majority \\
\hline \multicolumn{6}{|l|}{ System 2} \\
\hline Processor: Intel ${ }^{\circledR}$ Core $^{\mathrm{TM}}$ i7-9750H @ $2.6 \mathrm{GHz}$ & 4 & 0.6 & 40432 & 60 & Not \\
\hline Installed Memory: 32.00 GB & 5 & 0.28 & 41480 & 105 & And/or \\
\hline Operating System: Windows 10 & 6 & 0.57 & 42408 & 135 & Majority \\
\hline
\end{tabular}

a: Threads: Number of active threads;

b: Avg: Average percent of CPU consumption by the process (60 sec);

c: Private: The amount of physical memory in use by the process that cannot be used by other processes in KB 


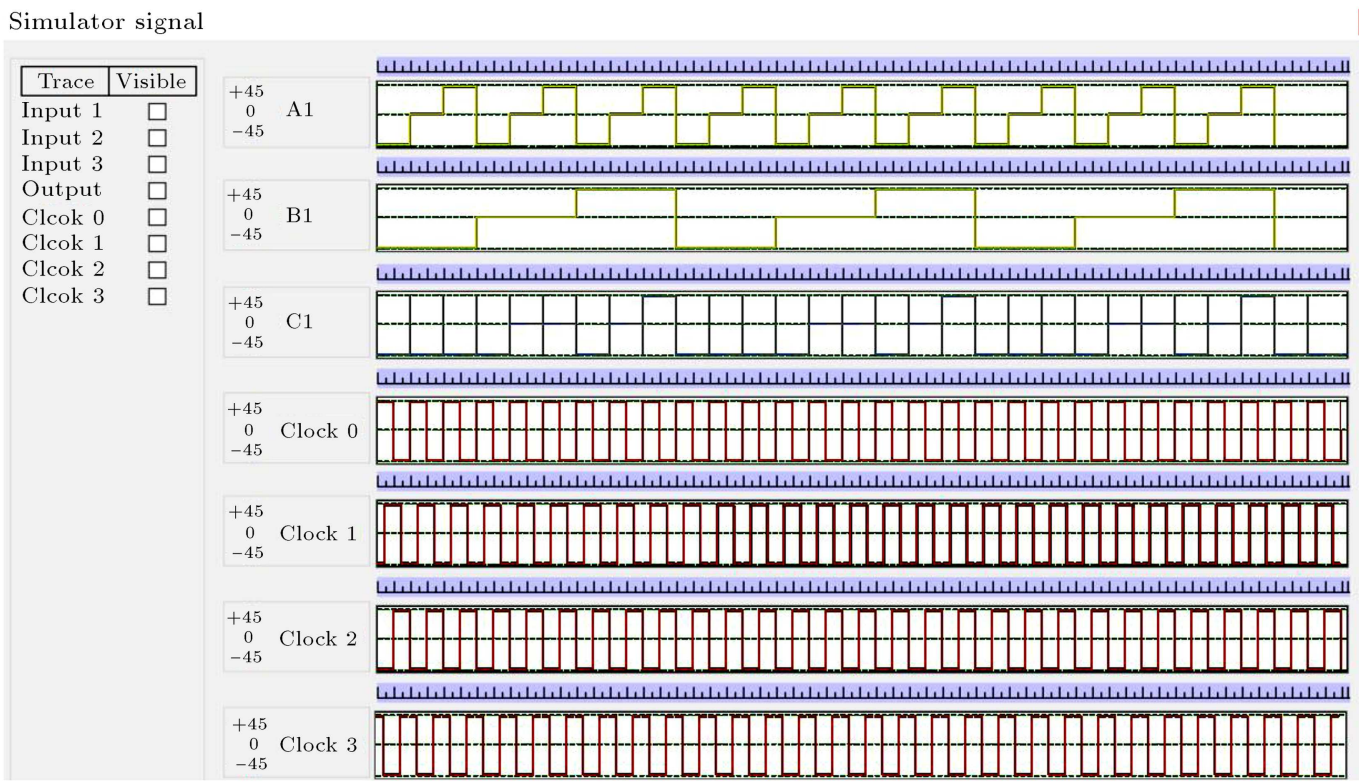

Figure 10. Simulation result of the sample circuit.

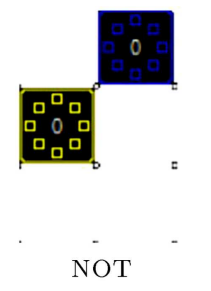

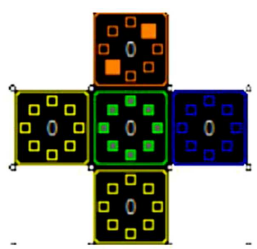

OR

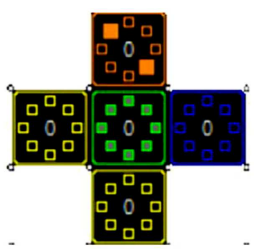

AND

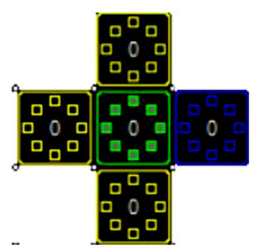

Majority

Figure 11. Primary ternary gates.

a very low CPU usage, even in an obsolete system. The amount of memory used is small enough.

\section{Future work}

Our QCA-team is constantly striving to offer comprehensive software for TQCA-systems designers. For this purpose, we redesigned the simulating-process and user interface for a faster and better startup in each version that seems necessary. The ability to customize layout size would appear in the next versions. Moreover, the ability to add more cell structures is under consideration. These features will allow users to simulate more intricate designs. We are trying to offer an improved tool for TQCA enthusiasts in every version of this software.

\section{Conclusion}

Every innovative concept needs a particular way to determine its functionality. TQCAsim is an accurate CAD tool that has been designed exclusively for ternary Quantum-dot Cellular Automata (QCA) logic designs and simulations. This tool has the ability to layout and verify any Ternary QCA (TQCA) systems. The simulation results are shown in graphical formats.
Although TQCAsim is currently in its infancy, great strides are made to enhance its usability and functionality in every version of this tool.

\section{Acknowledgement}

We are grateful to Milad Khani and Mohammad Amin Mianroodi for all their work and support.

\section{References}

1. Arulkarthick, V.J., Rathinaswamy, A., and Srihari, K. "Design of BCD adder with five input majority gate for QCA", Journal of Microprocessors and Microsystems, 75, 103040:1-9 (2020).

2. Cesar, T.F., Vieira, L.F.M., Vieira, M.A.M., et al. "Cellular automata-based byte error correction in QCA", Nano Communication Networks, 23, 100278:113 (2020).

3. Lent, C.S., Tougaw, P.D., Porod, W., et al. "Quantum cellular automata", Nanotechnology, 4(1), pp. 49-57 (1993).

4. Lent, C.S. and Tougaw, P.D. "A device architecture for computing with quantum dots", in Proc. IEEE, 85(4), pp. 541-547 (1997).

5. Lent, C.S., Isaksen, B., and Lieberman, M. "Molecular 
quantum-dot cellular automata", Journal of American Chemical Society, 125, pp. 1056-1063 (2003).

6. Lu, Y. and Lent, C.S. "Theoretical study of molecular quantum-dot cellular automata", Journal of Computational Electronics, 4(1), pp. 115-118 (2005).

7. Ahmed, S. and Naz, S.F. "Design of cost efficient modular digital QCA circuits using optimized XOR gate", in IEEE Transactions on Circuits and Systems II: Express Briefs (2020). DOI: 10.1109/TCSII.2020.3030180

8. Kianpour, M. and Sabbaghi-Nadooshan, R. "A novel quantum-dot cellular automata X-bit $\times 32$-bit SRAM", IEEE Trans. Very Large Scale Integration (VLSI) Systems, 24(3), pp. 827-836 (2016).

9. Kianpour, M. and Sabbaghi-Nadooshan, R. "A conventional design and simulation for CLB implementation of an FPGA quantum-dot cellular automata", Journal of Microprocessors and Microsystems, 38(8), pp. 1046-1062 (2014).

10. Kianpour, M. and Sabbaghi-Nadooshan, R. "A novel QCA implementation of MUX-based universal shift register", Journal of Computational Electronics, 13(1), pp. $198-210$ (2014).

11. Kianpour, M. and Sabbaghi-Nadooshan, R. "A novel design of 8-bit adder/subtractor by quantum-dot cellular automata", Journal of Computer and System Sciences, 80(7), pp. 1404-1414 (2014).

12. Babaie, S., Sadoghifar, A., and Bahar, A.N. "Design of an efficient multilayer arithmetic logic unit in quantum-dot cellular automata (QCA)", In IEEE Transactions on Circuits and Systems II: Express Briefs, 66(6), pp. 963-967 (2019).

13. Wang, L. and Xie, G. "A novel XOR/XNOR structure for modular design of QCA circuits", In IEEE Transactions on Circuits and Systems II: Express Briefs, 67(12), pp. 3327-3331 (2020).

14. Bajec, I.L., Zimic, N., and Mraz, M. "The ternary quantum-dot cell and ternary logic", Nanotechnology, 17(8), pp. 1937-1942 (2006).

15. Pecar, P., Mraz, M., Zimic, N., et al. "Solving the ternary QCA logic gate problem by means of adiabatic switching", Journal of Applied Physics, 47(6), pp. 5000-5006 (2008).

16. Tehrani, M.A., Bahrami, S., and Navi, K. "A novel ternary quantum-dot cell for solving majority voter gate problem", Applied Nanoscience, 4(3), pp. 255262 (2013).

17. Arjmand, M.M., Soryani, M., and Navi, K. "Coplanar wire crossing in quantum cellular automata using a ternary cell", IET Circuits, Devices \& Systems, 7(5), pp. 263-272 (2013).

18. Shahrom, E. and Hosseini, S.A. "A new low power multiplexer based ternary multiplier using CNTFETs", AEU - International Journal of Electronics and Communications, 93, pp. 191-207 (2018).

19. Daraei, A. and Hosseini, S.A. "Novel energy-efficient and high-noise margin quaternary circuits in nanoelectronics", AEU - International Journal of Electronics and Communications, 105, pp. 145-162 (2019).
20. Mohaghegh, S.M., Sabbaghi-Nadooshan, R., and Mohammadi, M. "Innovative model for ternary QCA gates", IET Circuits, Devices \& Systems, 12(2), pp. 189-195 (2018).

21. Mohaghegh, S.M., Sabbaghi-Nadooshan, R., and Mohammadi, M. "Designing ternary quantum-dot cellular automata logic circuits based upon an alternative model", Computers \& Electrical Engineering, 71, pp. 43-59 (2018).

22. Mohaghegh, S.M., Sabbaghi-Nadooshan, R., and Mohammadi, M. "Design of a ternary QCA multiplier and multiplexer: a model-based approach", Analog Integr Circ Sig Process, 101, pp. 23-29 (2019).

23. Almatrood, A.F. and Singh, H. "Design of generalized pipeline cellular array in quantum-dot cellular automata", IEEE Computer Architecture Letters, 17(1), pp. 29-32 (2018).

24. Abedi, D. and Jaberipur, G. "Decimal full adders specially designed for quantum-dot cellular automata", IEEE Trans. Circuits and Systems II: Express Briefs, 65(1), pp. 106-110 (2018).

25. Orlov, A.O., Amlani, I., Bernstein, G.H., et al. "Realization of a functional cell for quantum-dot cellular automata", Science, 277(5328), pp. 928-930 (1997).

26. Lent, C.S. and Isaksen, B. "Clocked molecular quantum-dot cellular automata", IEEE Transactions on Electron Devices, 50(9), pp. 1890-1896 (2003).

27. Amlani, I., Orlov, A.O., Kummamuru, R.K., et al. "Experimental demonstration of a leadless quantumdot cellular automata cell", Appl. Phys. Lett., 77(5), pp. $738-740$ (2000).

28. Das, K., De, D., and De, M. "Realisation of semiconductor ternary quantum dot cellular automata", IET Micro Nano Lett., 8(5), pp. 258-263 (2013).

29. Lu, Y., Liu, M., and Lent, C.S. "Molecular quantumdot cellular automata: From molecular structure to circuit dynamics", Journal of Applied Physics, 102(3), p. 034311 (2007).

30. Blair, E. "Electric-field inputs for molecular quantumdot cellular automata circuits", in IEEE Transactions on Nanotechnology, 18, pp. 453-460 (2019).

31. Alam, M.T., Siddiq, M.J., Bernstein, G.H., et al. "Onchip clocking for nanomagnet logic devices", IEEE Trans. Nanotechnology, 9(3), pp. 348-351 (2010).

32. http://www.qcasim.com

33. Pain, P., Sadhu, A., Das, K., et al. "Physical proof and simulation of ternary logic gate in ternary quantum dot cellular automata", Computational Advancement in Communication Circuits and Systems, Lecture Notes in Electrical Engineering, 575, pp. 375-385 (2020).

34. Bhoi, B.K., Misra, N.K., Dash, I., et al. "A redundant adder architecture in ternary quantum-dot cellular automata", Smart Intelligent Computing and Applications, 159, pp. 375-384 (2020).

35. Walus, K., Dysart, T.J., Jullien, G.A., et al. "QCADesigner: a rapid design and Simulation tool for quantum-dot cellular automata", IEEE Trans. Nanotechnology, 3(1), pp. 26-31 (2004). 


\section{Biographies}

Alireza Navidi was born in Tehran, Iran in 1992. He received the BSc degree in Electrical and Electronic Engineering from the University of Science and Culture, Tehran, Iran in 2015 and the MSc degree in the same field (with honors) from Islamic Azad University Central Tehran Branch, Tehran, Iran in 2017. He is currently pursuing a $\mathrm{PhD}$ degree, focusing on digital integrated circuits, logic gates, field-programmable gate array, quantum-dot cellular automata-based nanosystems design, and quantum computing at the Islamic Azad University Science and Research Branch, Tehran, Iran.

Reza Sabbaghi-Nadooshan received the BS and MS degrees from the University of Science and Technology, Tehran, Iran in 1991 and 1994, respectively, and obtained the PhD degree from Science and Research Branch, Islamic Azad University, Tehran in 2010, all in Electrical Engineering. He has been a Faculty Member with the Department of Electronics, Islamic Azad University Central Tehran Branch, Tehran since 1998. His current research interests include networks- on-chip, quantum-dot cellular automata, and nanocomputing.

Massoud Dousti received BS degree in Electrical Engineering from Orléans University, Orléans, France, and MS degree in Electronics (Microwave and Optics) from Limoges University, Limoges, France in 1991 and 1994 and $\mathrm{PhD}$ in Electronics (Active Microwave Circuits) from University of Paris VI, Pierre et Marie Currie in 1999. He served as a teaching assistant at the Department of Electrical Engineering at Ensea, Cergy Pontoise, France from 1998 to 2000. In 2001, he joined the Department of Electrical and Computer Engineering of Science and Research Branch, Islamic Azad University, Tehran, Iran, where he is now an Associate Professor. He is the author of eight books in the field of electronics and translated seven books from English to Farsi in the field of high-frequency, RF MEMS, and MMIC. He is the author and co-author of more than 105 published international journal and conference papers. His research interests are linear and non-linear RF/microwave/millimeter-wave circuits and systems design, high-frequency electronics (passive and active devices), RF MEMS, and MMIC technology. 